\title{
Finding Convex Sets Among Points in the Plane
}

D. Kleitman and L. Pachter

Department of Mathematics, MIT,

Cambridge, MA 02139, USA

\{djk,lpachter\}@math.mit.edu

Communicated by János Pach

Abstract. Let $g(n)$ denote the least value such that any $g(n)$ points in the plane in general position contain the vertices of a convex $n$-gon. In 1935, Erdős and Szekeres showed that $g(n)$ exists, and they obtained the bounds

$$
2^{n-2}+1 \leq g(n) \leq\left(\begin{array}{c}
2 n-4 \\
n-2
\end{array}\right)+1 .
$$

Chung and Graham have recently improved the upper bound by 1 ; the first improvement since the original Erdôs-Szekeres paper. We show that

$$
g(n) \leq\left(\begin{array}{c}
2 n-4 \\
n-2
\end{array}\right)+7-2 n .
$$

\section{Introduction}

Esther Klein, early in the 1930s, considered the following problem: Is it true that for every $n$, there is a least value $g(n)$, such that any set of $g(n)$ points in the plane in general position always contain the vertices of a convex $n$-gon?

Her question was partially resolved in a famous paper by Erdős and Szekeres [2] in which they showed that

$$
2^{n-2}+1 \leq g(n) \leq\left(\begin{array}{c}
2 n-4 \\
n-2
\end{array}\right)+1
$$

The upper bound was not improved for more than 50 years, which led Chung and Graham to raise the question: Can it be improved at all? They have recently shown [1] 

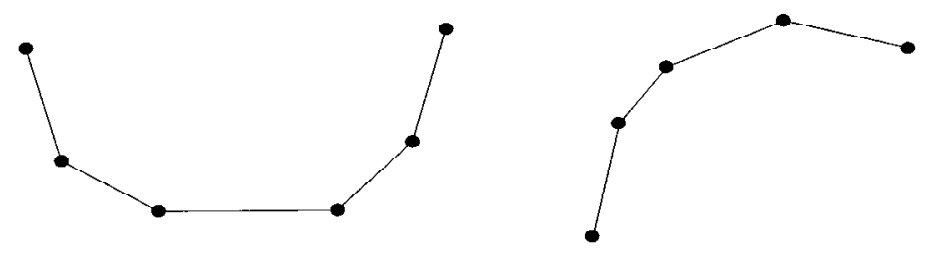

Fig. 1. A 6-cup and a 5-cap.

that it can be reduced by 1 . This modest improvement is like a drop of water that appears downstream from a dam. This drop can be the harbinger of a trickle, then perhaps a stream, and finally the dam may collapse with a rush of water.

It is the purpose of this paper to replace the Chung-Graham drop by a trickle. ${ }^{1}$

The original Erdős-Szekeres argument is based upon the notions of $n$-caps and $n$-cups:

Definition 1. An $n$-cap is a set of $n$ points which, when ordered from left to right, have the property that slopes of lines joining successive points are decreasing. Formally, an $n$-cap consists of $n$ points $\left(x_{1}, y_{1}\right),\left(x_{2}, y_{2}\right), \ldots,\left(x_{n}, y_{n}\right)$ satisfying $x_{1} \leq x_{2} \leq x_{3} \leq$ $\cdots \leq x_{n-1} \leq x_{n}$, and $\left(y_{i}-y_{i-1}\right) /\left(x_{i}-x_{i-1}\right)>\left(y_{i+1}-y_{i}\right) /\left(x_{i+1}-x_{i}\right)$ when $1<i<n$. An $n$-cup is defined in the same way except the slopes are required to be increasing.

Both $n$-caps and $n$-cups are special cases of convex polygons. They also have the property that if a single point is both the left endpoint of an $(n-1)$-cap and also the right endpoint of an $(m-1)$-cup, then either the cap or the cup can be extended by one point.

Erdős and Szekeres used this idea together with an induction argument to find the least number of points in the plane that always contain an $n$-cap or an $m$-cup:

Theorem 1 (Erdős-Szekeres). Let $f(n, m)$ be the least integer such that any $f(n, m)$ points in the plane in general position contain either an $n$-cap or an $m$-cup

$$
f(n, m)=\left(\begin{array}{c}
n+m-4 \\
n-2
\end{array}\right)+1
$$

We exploit the fact that the definition of caps and cups is dependent upon the orientation of the coordinate system used to describe the points in the plane. We show that if there are $\left(\begin{array}{c}n+m-4 \\ n-2\end{array}\right)+7-m-n$ points there is always an orientation of coordinates so that there is an $n$-cap or an $m$-cup. In particular, if we choose two consecutive vertices $a$ and $b$ from the convex hull of the points, and orient our coordinates so that the line segment $a b$ is vertical and forms the left end of the convex hull, then there is an $n$-cap or an $m$-cup in the configuration.

\footnotetext{
${ }^{1}$ Géza Tóth and Pavel Valtr have recently replaced our trickle with a stream by further improving the upper bound (see [5]).
} 


\section{Main Result}

We begin by introducing some terminology which we will find useful.

Definition 2. A configuration of points in general position is said to be vertical if the two leftmost points on the convex hull have the same horizontal coordinate.

Definition 3. For given $n, m$, a point in a configuration is said to be $a$-defective if it is neither the left endpoint of an $(n-1)$-cap nor the right endpoint of an ( $m-1)$-cup. Similarly, it is said to be $b$-defective if it is neither the right endpoint of an $(n-1)$-cap nor the left endpoint of an $(m-1)$-cup.

Theorem 2. Let $f_{V}(n, m)$ be the least integer such that any $f_{V}(n, m)$ points in a vertical configuration contain an n-cap or an m-cup.

$$
f_{V}(n, m)=\left(\begin{array}{c}
n+m-4 \\
n-2
\end{array}\right)+7-m-n .
$$

Proof of the Upper Bound. We argue by induction. First, notice that if $m$ or $n$ are 3 we have $f_{V}(n, 3)=f_{V}(3, m)=3$. So assume that $m, n \geq 4$.

Consider a vertical configuration, $X$, of $f_{V}(n, m)-1$ points with no $n$-caps or $m$-cups. Let $a$ and $b$ be the two leftmost endpoints in $X$, chosen so that $a$ is above $b$ (see Fig. 2).

Our argument is based upon two observations: First, note that since $a$ and $b$ are on the left of our configuration, they cannot be right endpoints of $(n-1)$-caps or $(m-1)$-cups. We can also say that $a$ is not the left endpoint of an $(n-1)$-cap because if it were we could use $b$ to extend it to an $n$-cap. Similarly, $b$ is not the left endpoint of an $(m-1)$-cup. It follows that $a$ is $a$-defective and $b$ is $b$-defective (the Chung-Graham theorem follows immediately from this observation).

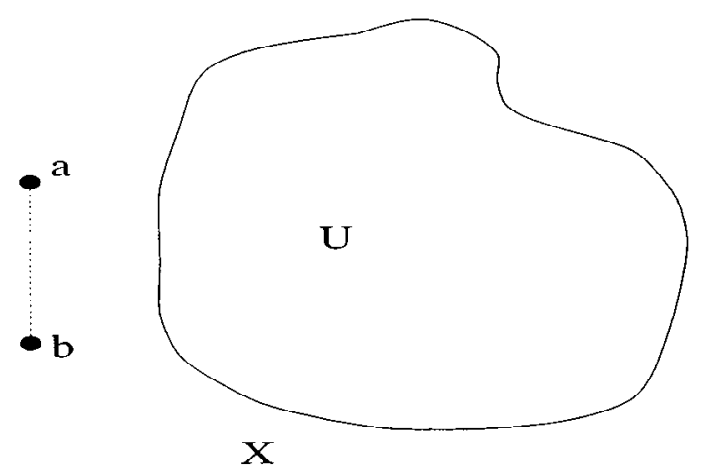

Fig. 2. A diagram of the configuration $X$.

Notes. $\quad 1 . a$ and $b$ form a vertical line.

2. All other points are in $U$ to the right of $a b$.

3. Any cap with left endpoint $a$ extends to a larger one ending at $b$.

4. Any cup with left endpoint $b$ extends to a larger one ending at $a$. 
Second, we can use the original Erdős-Szekeres argument for our specific orientation of points. We build a set $R$ of points consisting of right endpoints of ( $m-1)$-cups. We do this by finding an $(m-1)$-cup, adding the right endpoint to $R$, removing the point from $X$ and repeating the procedure. Notice that as we do this $X$ remains a vertical configuration. Since there are no $n$-caps in $X, R$ contains $f_{V}(n, m)-f_{V}(n, m-1)$ points. We can also add the point $a$ to $R$ since it is $a$-defective. We therefore find $f_{V}(n, m)-f_{V}(n, m-1)+1$ points which cannot contain an $(n-1)$-cap or an $m$-cup. We have a contradiction if

$$
f_{V}(n, m)-f_{V}(n, m-1)+1 \geq f(n-1, m)
$$

in which case the set $R$ would have an $(n-1)$-cap or $m$-cup.

We can also build a set consisting of $f_{V}(n, m)-f_{V}(n, m-1)$ right endpoints of $(n-1)$-caps together with the point $b$. We therefore have the recursions:

$$
\begin{aligned}
& f_{V}(n, m) \leq f(n, m-1)+f_{V}(n-1, m)-2, \\
& f_{V}(n, m) \leq f(n-1, m)+f_{V}(n, m-1)-2 .
\end{aligned}
$$

These recursions, together with the known values of $f(n, m)$ and the boundary conditions when the smaller argument is 3 , have the solution:

$$
f_{V}(n, m) \leq\left(\begin{array}{c}
n+m-4 \\
n-2
\end{array}\right)+7-m-n
$$

Given a configuration of points, we can rotate it so that it is vertical. Setting $m=n$ in (7), we have

\section{Corollary 1.}

$$
g(n) \leq\left(\begin{array}{c}
2 n-4 \\
n-2
\end{array}\right)+7-2 n
$$

Proof of the Lower Bound. We will show that the two recursions (5) and (6) are in fact equalities. Assume that $n, m \geq 4$. Let $S_{1}$ be a maximal vertical configuration with no $(n-1)$-cap or $m$-cup, and let $S_{2}$ be a maximal configuration (not necessarily vertical) with no $n$-cap or $(m-1)$-cup. The cardinality of $S_{1}$ will be $f_{V}(n-1, m)-1$, and the cardinality of $S_{2}$ will be $f(n, m-1)-1$. Choose $S_{2}$ so that the leftmost point is also the top point (this can always be arranged, see [4]).

Construct a vertical configuration as follows:

1. Transform $S_{1}$ and $S_{2}$ by affine transformations so that $S_{1}$ lies along the $x$-axis and $S_{2}$ along the line $y=-x$.

2. The leftmost point of $S_{1}$ (with smaller $y$-coordinate) coincides with the leftmost point of $S_{2}$. Call this point $p$.

3. The $x$-coordinates of points of $S_{1}$ are smaller than those of $S_{2}-p$.

4. $S_{1}-p$ lies above each line determined by points of $S_{2}$.

5. $S_{2}-p$ lies above each line determined by points of $S_{1}$. 


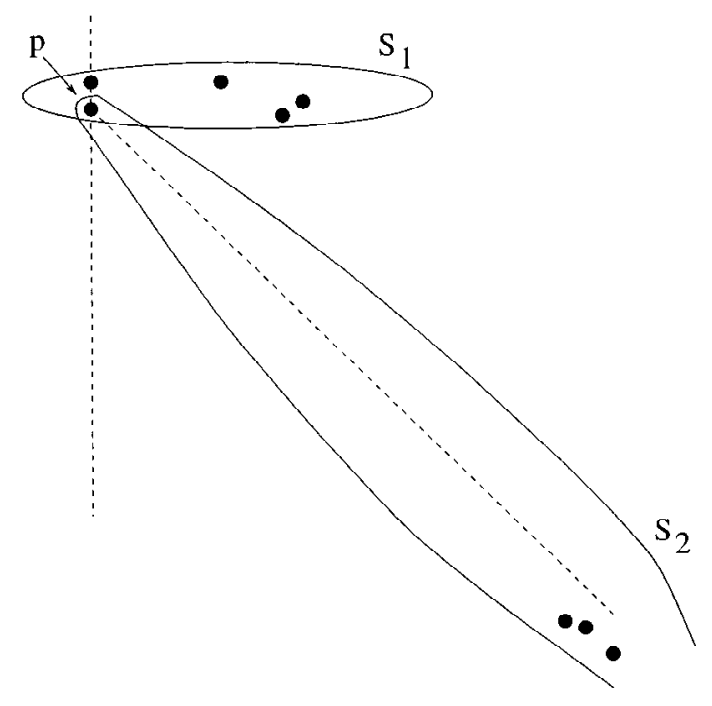

Fig. 3. Construction of a vertical configuration.

No $(n-2)$-cap in $S_{1}$ can be extended by more than 1 using a point in $S_{2}$. Similarly, no $(n-2)$-cup in $S_{2}$ can be extended by more than 1 using a point in $S_{1}$. Thus we see that our point set contains no $n$-caps or $m$-cups. Furthermore, the size of our vertical configuration implies that

$$
f_{V}(n, m) \geq f(n, m-1)+f_{V}(n-1, m)-2 .
$$

The above construction can be modified to place $S_{2}$ above $S_{1}$ along the line $y=x$, yielding

$$
f_{V}(n, m) \geq f(n-1, m)+f_{V}(n, m-1)-2 .
$$

\section{Comments}

What propels the argument of the last section is the observation that $a$ and $b$ must be defective under the given orientation. Furthermore, $a$ and $b$ remain defective even after the removal of right endpoints of $(n-1)$-caps and $(m-1)$-cups.

A point that is the left or right endpoint of an $(n-1)$-cap or $(m-1)$-cup, can, upon rotating the coordinate system, become an $a$-defective or $b$-defective point. This suggests that there may be angles at which there are many defective points. A careful analysis of the conditions under which a point is defective, coupled with the above observation, may allow an improvement in the upper bound for $g(n)$. 


\section{Acknowledgments}

We thank Géza Tóth and Pavel Valtr for contributing the lower bound construction. We also thank the referee for numerous helpful suggestions and comments.

\section{References}

1. F. R. K. Chung and R. L. Graham, Forced convex $n$-gons in the plane, Discrete Comput. Geom., this issue, pp. 367-371.

2. P. Erdős and G. Szekeres, A combinatorial problem in geometry, Compositio Math., 2 (1935), 463-470.

3. P. Erdős and G. Szekeres, On some extremum problems in elementary geometry, Ann. Univ. Sci. Budapest. Eötvös Sect. Math., 3-4 (1961), 53-62.

4. L. Lovász, Combinatorial Problems and Exercises, North-Holland, Amsterdam, 1979, §14(30), pp. 501503.

5. G. Tóth and P. Valtr, Note on the Erdős-Szekeres theorem, Discrete Comput. Geom., this issue, pp. 457-459.

Received January 1, 1997, and in revised form June 6, 1997. 